\title{
Multicell CDMA Network Design
}

\author{
Robert G. Akl, Member, IEEE, Manju V. Hegde, Member, IEEE, Mort Naraghi-Pour, Member, IEEE, and \\ Paul S. Min, Senior Member, IEEE
}

\begin{abstract}
Traditional design rules for cellular networks are not directly applicable to code division multiple access (CDMA) networks where intercell interference is not mitigated by cell placement and careful frequency planning. For transmission quality requirements, a minimum signal-to-interference ratio (SIR) must be achieved. The base-station location, its pilot-signal power (which determines the size of the cell), and the transmission power of the mobiles all affect the received SIR. In addition, because of the need for power control in CDMA networks, large cells can cause a lot of interference to adjacent small cells, posing another constraint to design. In order to maximize the network capacity associated with a design, we develop a methodology to calculate the sensitivity of capacity to base-station location, pilot-signal power, and transmission power of each mobile. To alleviate the problem caused by different cell sizes, we introduce the power compensation factor, by which the nominal power of the mobiles in every cell is adjusted. We then use the calculated sensitivities in an iterative algorithm to determine the optimal locations of the base stations, pilot-signal powers, and power compensation factors in order to maximize capacity. We show examples of how networks using these design techniques provide higher capacity than those designed using traditional techniques.
\end{abstract}

Index Terms-Capacity optimization, cell design, code division multiple access (CDMA), location design, power compensation.

\section{INTRODUCTION}

$\mathbf{T}$ HE reverse link capacity of a single cell in a cellular code division multiple access (CDMA) network depends on the interference of users within that cell (intracell interference), as well as on the interference of users in adjacent cells (intercell interference) [1]-[3]. Thus, the number of simultaneous users that can be handled within one cell depends on the number of simultaneous users in all the cells in the network. This interference limitation makes the cell placement design in CDMA networks particularly difficult: the problem of placing cells in a region with a given user profile would require the calculation of the intercell interference, which depends on the cell geometry, the transmit power levels of the users, and the number of users in adjacent cells. This problem is not present in networks that use fixed channel assignment algorithms, wherein cochannel interference is eliminated by using different frequency sets in adjacent cells, thereby separating the problem of cell placement and cell capacity. In such networks, the design rule-of-thumb is to place cells so that each will have a constant demand. Thus,

Manuscript received November 30, 1999; revised July 26, 2000. This work was supported in part by the McDonnell Foundation and in part by the National Science Foundation under Grant NCR 9706545.

R. G. Akl, M. V. Hegde, and P. S. Min are with the Department of Electrical Engineering, Washington University, St. Louis, MO 63130 USA.

M. Naraghi-Pour is with the Department of Electrical and Computer Engineering, Louisiana State University, Baton Rouge, LA 70803 USA.

Publisher Item Identifier S 0018-9545(01)03959-7. smaller cells are used in areas of high demand while larger cells are used in areas of low demand. In CDMA networks, differing usage results in differing intercell interference, which suggests the possibility of more efficient topology design. In this paper, we describe a new network topology design technique for CDMA networks that outperforms traditional techniques.

In [3]-[12], the authors calculate the capacity of a single CDMA cell or a CDMA network by restricting analysis to reverse link capacity. These papers concede that CDMA networks are interference- and reverse-link limited in that the system capacity for the reverse link will be lower than that of the forward link. In [1], the authors analyze both forward- and reverse-link capacity. They have shown that for an ideal power control and hard handoff case, reverse-link capacity limits the system capacity; however, the difference between forward- and reverse-link capacities is not large. In [13], the authors show that reverse-link capacity is increased considerably by soft handoff, but, at the same time, imperfect power control reduces it and compensates for the increase. On the other hand, forward-link capacity is decreased due to soft handoff, and the reduction is shown to be more than the difference between reverse- and forward-link capacities. They believe that the overload in forward link limits the capacity of the system. From the above discussion, it is evident that among researchers, a consensus does not exist on whether the CDMA system capacity is reverse- or forward-link limited. However, the majority of the literature published on the subject is of the former view. In light of this, in this paper we consider the reverse-link capacity only. In the rest of this paper, when we refer to capacity, we actually mean the reverse-link capacity.

Despite the abundant literature on CDMA, few contributions address the cell design problem. The main emphasis to date has been on capacity analysis through simulation with uniform cells. In [14], the performance of IS-95-based CDMA systems [15] is studied in nonuniform and uniform traffic distribution with equal cell sizes. The elements that are investigated are the pilot channel chip energy to received signal power spectral density ratio, the forward link bit energy to noise power spectral density ratio, and the reverse link bit energy to noise power spectral density ratio. Monte Carlo simulations are carried out under a hexagonal omnicell configuration to obtain statistics of capacity, handoff percentage, and signal and interference levels.

In [16], the problem of nonuniform traffic's decreasing of the system capacity in microcell CDMA systems is investigated. The authors propose a scheme whereby every base station adaptively controls the required transmission power level of the mobiles based on minimizing the difference between the target signal-to-interference ratio (SIR) and the observed SIR on the 
reverse link at the base station. The authors evaluate the effectiveness of their proposed method with regard to the signal quality and the outage probability through computer simulation. The results show that the proposed method is effective against localized high-density traffic, but the effectiveness becomes smaller as the traffic load in the vicinity of congested cells increases.

In [8] and [9], an iterative algorithm for cell design that minimizes the disparity in communication quality between base stations is proposed. To equalize the SIR in order to maximize capacity, every base station adjusts both the pilot-signal power and the desired transmission power level based on the difference between the average SIR and the observed SIR on the reverse link. The authors confirm the effectiveness of the proposed algorithm through computer simulation.

In [11], the problem of adaptive cell sectorization to increase capacity in CDMA systems is investigated. The authors propose to minimize the total received power and the total transmit power of the mobiles in order to reduce intercell interference while retaining acceptable quality of service. The results show that under nonuniform traffic conditions, the optimum arrangement of the sector boundaries is quite different from uniform cell sectorization.

In a CDMA network, the near-far problem necessitates power control, whereby the transmit power of mobiles is proportional to distance (from the base station) raised to the path-loss exponent. Typically, the power control scheme used in CDMA networks is signal level based, i.e., the power control equalizes the received power from the mobiles at the base station. We assume such a power control scheme in this paper. In particular, SIR-based power control is not investigated [17] [18]. When large cells are adjacent to small cells, users at the boundaries of large cells cause a lot of interference to users in small cells. This causes a significant reduction in the capacity of the small cells. To alleviate this problem, we propose to adjust the nominal power of the mobiles in every cell by a power compensation factor (PCF) [19]. Since CDMA is interference limited, any decrease in the amount of interference translates into a capacity gain. Increasing the pilot-signal power of a base station increases the coverage region of that cell and thus increases the number of users and the intracell interference in that cell. However, it will decrease the number of users in the adjacent cells, thus decreasing the intercell interference on this base station. In addition, changing the location of a base station changes the coverage region of that cell and the coverage regions of the adjacent cells. Thus, by controlling the transmitted pilot-signal power and adjusting the location of the base stations, the coverage region of each cell is controlled, which in turn controls the intracell and intercell interference. Given a fixed configuration of user distribution, we try to place a given number of cells in order to maximize capacity. We evaluate the capacity of the entire network as a function of all the PCFs, the base-station locations, and the transmitted pilot-signal powers and present an optimization framework that allows us to maximize capacity. We develop design rules that apply to general user configurations (uniform or with hot spots). We validate our design rules by presenting comparative capacity results for networks that are designed by our method versus those designed with traditional rules.

The remainder of this paper is organized as follows. In Section II, we calculate the relative average intercell interference. In Section III, we define network capacity. In Section IV, we study the sensitivity of the network capacity with respect to base-station locations, pilot-signal powers, and power compensation factors. In Section V, the optimization of capacity is performed, and numerical results are presented in Section VI. Section VII concludes this paper.

\section{RELATIVE AVERAGE INTERCELL INTERFERENCE}

Consider two cells $i$ and $j$. We assume that each user is always communicating with and is power controlled by the base station that has the highest received power at the user. Let $C_{j}$ denote the region where the received pilot-signal power from base station $j$ is the highest among all base stations. A user located at coordinates $(x, y)$ is at distance $r_{j}(x, y)$ from base station $j$. Let $n_{j}$ be the number of users in cell $j$ and $A_{j}=\operatorname{Area}\left(C_{j}\right)$, the area of cell $j$. It is assumed that the power-control mechanism overcomes both large-scale path loss and shadow fading. It does not, however, overcome the fast fluctuations of the signal power associated with Rayleigh fading [1]. The propagation loss of a user in cell $j$ is modeled as the product of the $m$ th power of distance and a log-normal component representing shadowing losses. Now let $\chi_{i}$ denote the Rayleigh random variable that represents the fading on the path from this user to cell $i$. The average of $\chi_{i}^{2}$ is the log-normal fading on that path, i.e., $E\left[\chi_{i}^{2} \mid \zeta_{i}\right]=10^{-\zeta_{i} / 10}$ [20], where $\zeta_{i}$ is the decibel attenuation due to shadowing and is a Gaussian random variable with zero mean and standard deviation $\sigma_{s}$. Consequently, the relative average interference at cell $i$ caused by all users in cell $j$ is given by [21]

$$
I_{j i}=E\left[\iint_{C_{j}} \frac{r_{j}^{m}(x, y) 10^{\zeta_{j} / 10}}{r_{i}^{m}(x, y) / \chi_{i}^{2}} \frac{n_{j}}{A_{j}} d A(x, y)\right] .
$$

The expectation is calculated as follows:

$$
\begin{aligned}
\mathrm{E}\left[10^{\zeta_{j} / 10} \cdot \chi_{i}^{2}\right] & =\mathrm{E}\left[\mathrm{E}\left[10^{\zeta_{j} / 10} \cdot \chi_{i}^{2} \mid \zeta_{i}, \zeta_{j}\right]\right] \\
& =\mathrm{E}\left[10^{\zeta_{j} / 10} \mathrm{E}\left[\chi_{i}^{2} \mid \zeta_{i}, \zeta_{j}\right]\right] \\
& =\mathrm{E}\left[10^{\zeta_{j} / 10} \cdot 10^{-\zeta_{i} / 10}\right] .
\end{aligned}
$$

Let $x=\zeta_{j}-\zeta_{i}$, where $x$ is a Gaussian random variable with zero mean and variance equal to $2 \sigma_{s}^{2}$ since $\zeta_{j}$ and $\zeta_{i}$ are independent. Substituting in (2), we get

$$
\begin{aligned}
\mathrm{E}\left[10^{\left(\zeta_{j}-\zeta_{i}\right) / 10}\right] & =\mathrm{E}\left[e^{\gamma x}\right]=\int_{-\infty}^{+\infty} \frac{e^{\gamma x} e^{x^{2} / 4 \sigma_{s}^{2}}}{\sqrt{4 \pi \sigma_{s}^{2}}} d x \\
& =e^{\left(\gamma \sigma_{s}\right)^{2}}
\end{aligned}
$$

where $\gamma=\ln (10) / 10$. Substituting the result back into (1)

$$
I_{j i}=e^{\left(\gamma \sigma_{s}\right)^{2}} \frac{n_{j}}{A_{j}} \iint_{C_{j}} \frac{r_{j}^{m}(x, y)}{r_{i}^{m}(x, y)} d A(x, y) .
$$


Let $\kappa_{j i}$ denote the per-user intercell interference factor of cell $j$ to cell $i$, i.e., $\kappa_{j i}=I_{j i} / n_{j}$. Note that in our model, $\kappa_{i i}$ equals zero.

Equation (4) is used to calculate the relative average intercell interference for a uniform user distribution, i.e., when the relative user density at $(x, y)$ in cell $j$ is $n_{j} / A_{j}$. For a nonuniform user distribution, let $\omega(x, y)$ be the relative user density at $(x, y)$. A hot spot is a region of a cell with a higher relative user density than the rest of the cell. The relative average intercell interference at cell $i$ caused by all users from cell $j$ for the general case becomes

$$
I_{j i}=e^{\left(\gamma \sigma_{s}\right)^{2}} \iint_{C_{j}} \frac{r_{j}^{m}(x, y)}{r_{i}^{m}(x, y)} \omega(x, y) d A(x, y)
$$

The $I_{j i} \mathrm{~s}$ will be used to determine the reverse-link capacity. A closed-form expression can be derived for $I_{j i}$ for the case of a uniform user distribution and a specific cell geometry (e.g., hexagonal). However, since for the case of nonuniform user distribution we evaluate $I_{j i}$ in (5) numerically, we have used the same approach for the uniform case and have not obtained the closed-form solution for (4).

\section{CDMA NETWORK CAPACITY}

Consider a multicell CDMA network with spread signal bandwidth of $W$, information rate of $R$ bits/s, voice activity factor of $\alpha$, and background noise spectral density of $N_{0}$. Assuming a total of $M$ cells with $n_{i}$ users in cell $i$, the bit energy to interference density ratio in cell $i$ is given by [22]

$$
\begin{gathered}
\left(\frac{E_{b}}{I_{0}}\right)_{i}=\frac{E_{b}}{\alpha\left(R E_{b}\right)\left(n_{i}-1+\sum_{j=1}^{M} n_{j} \kappa_{j i}\right) / W+N_{0}}, \\
\text { for } i=1, \ldots, M .
\end{gathered}
$$

To achieve a required bit error rate we must have $\left(E_{b} / I_{0}\right)_{i} \geq \Gamma$ for some constant $\Gamma$. Thus, rewriting (6), the number of users in every cell must satisfy

$$
\begin{gathered}
n_{i}+\sum_{j=1}^{M} n_{j} \kappa_{j i} \leq \frac{W / R}{\alpha}\left(\frac{1}{\Gamma}-\frac{1}{E_{b} / N_{0}}\right)+1 \triangleq c_{\mathrm{eff}} \\
\text { for } i=1, \ldots, M .
\end{gathered}
$$

A set of users $\underline{n}=\left(n_{1}, \ldots, n_{M}\right)$ satisfying the above equations is said to be a feasible user configuration, i.e., one that satisfies the $E_{b} / I_{0}$ constraint. The right-hand side of (7) is a constant, determined by system parameters and by the desired maximum bit error rate, and can be regarded as the total number of effective channels $c_{\text {eff }}$ available to the system. As can be seen from (7), the capacities of the CDMA cells in a network must be considered jointly. Thus the notion of capacity in a CDMA network is that of a capacity region, which indicates all tradeoffs in capacity between the cells in the network.
We define equal capacity as the requirement that all cells have an equal number of users, i.e., $n_{i}=n$ for all $i$. For the equal capacity case, the network capacity $N$ is equal to $M n$, where

$$
n=\min _{1 \leq i \leq M}\left[\frac{c_{\mathrm{eff}}}{1+\sum_{j=1}^{M} \kappa_{j i}}\right] .
$$

In general, for a given fixed configuration of user distribution, a feasible user configuration yields a network capacity $N$ that is the solution to the following optimization problem

$$
\begin{aligned}
\max _{\underline{n}} & \sum_{i=1}^{M} n_{i} \\
\text { subject to } & n_{i}+\sum_{j=1}^{M} n_{j} \kappa_{j i} \leq c_{\mathrm{eff}}, \\
& \text { for } i=1, \ldots, M .
\end{aligned}
$$

We should point out that the pilot-signal strengths and the basestation locations affect the values of the set of factors $\kappa_{j i}$ and thereby affect the decision variables $n_{i}$ in (9).

The optimization problem in (9) is an integer programming (IP) problem. One technique to solve the IP problem is based on dividing the problem into a number of smaller problems in a method called branch and bound [23]. Branch and bound is a systematic method for implicitly enumerating all possible combinations of the integer variables in a model. The number of subproblems and branches required can become extremely large.

If the integer variables $n_{i}, i=1, \ldots, M$, are relaxed and assumed to be continuous variables, then (9) becomes a linear programming (LP) problem whose solution can be obtained by any general LP technique, e.g., the simplex method [24]. We should point out that (9) is a convex optimization problem, and therefore, the approach described above does converge to a global optimal solution. It should also be noted that although the optimization problem in (9) maximizes the total network capacity and provides an improvement over that obtained from (8), the capacity of individual cells that results from (9) may vary significantly.

In Section VI, we provide results to the continuous relaxation of (9), which give an upper bound on the optimal value. We give the rounded-down solution (rounding down the solution of the continuous relaxation problem), which in general is not the optimal solution of the IP problem. We also solve the IP problem and provide the optimal solution as well as the number of branches required to arrive at the solution.

\section{SENSITIVITY ANALYSIS}

Having formulated the optimization problem that calculates network capacity, we now investigate the effect of changing the transmission power of the mobiles, the pilot-signal powers, and the locations of the base stations on this capacity. Consider a network with a large cell $j$ adjacent to a small cell $i$, and consider two users $A$ and $B$ both located at the boundary of these two cells as shown in Fig. 1. User $A$ will cause a lot of interference to 


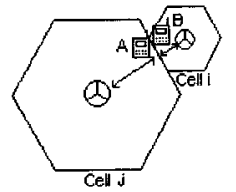

Fig. 1. Effect of a large cell adjacent to a small cell.

user $B$ because mobiles' transmit power is proportional to their distance from the base station raised to the path-loss exponent. Thus, a small cell adjacent to a large cell will experience a great deal of interference - which causes a significant reduction in the capacity of the small cell.

This problem can be resolved by adjusting the nominal power of the mobiles in every cell by the PCF in order to make the SIR in small cells comparable to that in large cells. If cell $j$ has a PCF $\beta_{j}$, its mobiles' signal powers have increased by a factor of $\beta_{j}$. Thus, the new relative average intercell interference of cell $j$ to cell $i$ becomes

$$
I_{j i}=e^{\left(\gamma \sigma_{s}\right)^{2}} \iint_{C_{j}} \frac{\beta_{j} r_{j}^{m}(x, y)}{r_{i}^{m}(x, y)} \frac{n_{j}}{A_{j}} d A(x, y)
$$

As a result, the new intercell interference factor per user becomes $\beta_{j} \kappa_{j i}$. Thus, once the original intercell interference factors have been calculated (for PCFs equal to one), changing the PCFs for the cells does not require recalculation of the original $\kappa_{j i}$ since $I_{j i}$ is linear in $\beta_{j}$.

Let $S$ be the received nominal power at base station $i$ without PCF. Then, $S=R E_{b}$. With PCF, the received power at base station $i$ is $\beta_{i} S$. Now, $\beta_{i} S=R\left(E_{b}^{\prime}\right)_{i}$. The ratio of bit energy to interference density is

$$
\begin{aligned}
\left(\frac{E_{b}^{\prime}}{I_{0}^{\prime}}\right)_{i}= & \frac{\beta_{i} S / R}{\alpha\left(n_{i}-1\right) \beta_{i} S / W+\alpha \sum_{j=1}^{M} n_{j} \kappa_{j i} \beta_{j} S / W+N_{0}} \\
= & \frac{W / R}{\alpha\left(n_{i}-1\right)+\alpha \sum_{j=1}^{M} n_{j} \kappa_{j i} \frac{\beta_{j}}{\beta_{i}}+\frac{N_{0} W}{\beta_{i} S}} \\
= & \frac{W / R}{\alpha\left(n_{i}-1\right)+\alpha \sum_{j=1}^{M} n_{j} \kappa_{j i} \frac{\beta_{j}}{\beta_{i}}+\frac{N_{0} W}{\beta_{i} R E_{b}}} \\
= & \frac{W / R}{\left[n_{i}-1+\sum_{j=1}^{M} n_{j} \kappa_{j i} \frac{\beta_{j}}{\beta_{i}}\right]+\frac{W / R}{\beta_{i} E_{b} / N_{0}}}
\end{aligned}
$$

To achieve a required bit error rate, we must have $\left(E_{b}^{\prime} / I_{0}^{\prime}\right)_{i} \geq \Gamma$, yielding

$$
\frac{W / R}{\alpha\left[n_{i}-1+\sum_{j=1}^{M} n_{j} \kappa_{j i} \frac{\beta_{j}}{\beta_{i}}\right]+\frac{W / R}{\beta_{i} E_{b} / N_{0}}} \geq \Gamma .
$$

Rearranging terms

$$
\begin{aligned}
n_{i}+\sum_{j=1}^{M} n_{j} \kappa_{j i} \frac{\beta_{j}}{\beta_{i}} & \leq \frac{W / R}{\alpha}\left(\frac{1}{\Gamma}-\frac{1}{\beta_{i} E_{b} / N_{0}}\right)+1 \\
& \triangleq c_{\mathrm{eff}}^{(i)}
\end{aligned}
$$

Let

$$
c_{i}=n_{i}+\sum_{j=1}^{M} n_{j} \frac{\beta_{j} \kappa_{j i}}{\beta_{i}}-c_{\mathrm{eff}}^{(i)}, \quad \text { for } i=1, \ldots, M .
$$

Assuming that the variables $n_{i}, i=1, \ldots, M$, are relaxed to be continuous variables, the derivative of $c_{i}$ with respect to $n_{k}$ is

$$
\frac{\partial c_{i}}{\partial n_{k}}= \begin{cases}1, & \text { if } k=i \\ \frac{\beta_{k} \kappa_{k i}}{\beta_{i}}, & \text { if } k \neq i\end{cases}
$$

The above derivatives will be used in the solution to the optimization problems (31)-(34).

\section{A. Sensitivity with Respect to Power Compensation Factors}

Increasing the PCF of a cell increases the SIR of that cell, thereby increasing the capacity of that cell. On the other hand, it will also increase the interference into its adjacent cells, thereby reducing the capacity of those cells. Therefore, we wish to find the optimal values for the PCFs that will maximize the capacity of the entire network. The PCFs also provide flexibility in the allocation of capacity. By changing the PCFs, capacity can be exchanged between cells. Our approach is to first calculate the derivatives of the network capacity with respect to the PCFs and to use them in an optimization algorithm. These derivatives capture the effect of increases in the PCF of one cell on the capacity of the entire network.

Combining (8) and (13), the equal capacity case becomes

$$
n=\min _{1 \leq i \leq M}\left[\frac{\beta_{i} c_{\mathrm{eff}}^{(i)}}{\beta_{i}+\sum_{j=1}^{M} \beta_{j} \kappa_{j i}}\right] .
$$

Let $i^{*}$ denote the index that minimizes (16). For numerical purposes, we allow $n$ to be a real number. The derivative of $n$ with respect to a $\mathrm{PCF} \beta_{k}$ is

$$
\frac{\partial n}{\partial \beta_{k}}= \begin{cases}\frac{\left(\beta_{i^{*}} c_{\mathrm{eff}}^{\left(i^{*}\right)}+\psi\right) \sum_{j=1}^{M} \beta_{j} \kappa_{j i^{*}}+\beta_{i^{*}} \psi}{\beta_{i^{*}}\left(\beta_{i^{*}}+\sum_{j=1}^{M} \beta_{j} \kappa_{j i^{*}}\right)^{2}}, & \text { if } k=i^{*} \\ \frac{-\beta_{i^{*}} c_{\mathrm{eff}}^{\left(i^{*}\right)} \kappa_{k i^{*}}}{\left(\beta_{i^{*}}+\sum_{j=1}^{M} \beta_{j} \kappa_{j i^{*}}\right)^{2}}, & \text { if } k \neq i^{*}\end{cases}
$$

where $\psi=W / R / \alpha E_{b} / N_{0}$. 
For the general case where the capacity in each cell may be different, the derivative of $c_{i}$ with respect to $\beta_{k}$ is

$$
\frac{\partial c_{i}}{\partial \beta_{k}}= \begin{cases}-\sum_{j=1}^{M} n_{j} \frac{\beta_{j} \kappa_{j k}}{\beta_{k}^{2}}-\frac{\psi}{\beta_{k}^{2}}, & \text { if } k=i \\ \frac{n_{k} \kappa_{k i}}{\beta_{i}}, & \text { if } k \neq i\end{cases}
$$

The above derivatives will be used in the solution to the optimization problems (31) and (34).

\section{B. Sensitivity with Respect to Transmitted Pilot-Signal Power}

In a CDMA network, it is important to control the intercell interference. Increasing the pilot-signal power of a base station expands the coverage area of that cell, thereby increasing the number of users in that cell and thus the intracell interference. On the other hand, it will decrease the number of users in the adjacent cells, thus decreasing the intercell interference on this base station. The opposite effect takes place in the adjacent cells. The intercell interference into these cells increases and the intracell interference decreases. Therefore, we wish to find the optimal values of the transmitted pilot-signal powers that will maximize the capacity of the entire network. Let $L_{i}^{(x)}$ and $L_{i}^{(y)}$ be the coordinates of base station $i$ and $T_{i}$ the transmitted pilot-signal power of base station $i$. For brevity, we use the notation $L_{i}=\left(L_{i}^{(x)}, L_{i}^{(y)}\right)$. Let $\mathcal{A}_{i}$ denote the set of base stations adjacent to base station $i$. Given a path-loss model, the region of cell $i, C_{i}$, is completely determined by $L_{i}^{(x)}, L_{i}^{(y)}, L_{j}^{(x)}, L_{j}^{(y)}$, $T_{i}$, and $T_{j}$ for $j \in \mathcal{A}_{i}$.

For example, consider two adjacent cells $i$ and $j$. Let $b_{i j}$ be the point on the straight line connecting $L_{i}$ and $L_{j}$, where the received pilot power from base station $i$ equals the received pilot power from base station $j$. Then, using the COST-231 model for path loss [25]

$$
b_{i j}=\frac{e^{\delta\left(T_{i}-T_{j}\right)}}{1+e^{\delta\left(T_{i}-T_{j}\right)}} d_{i j}
$$

where $d_{i j}$ is the distance between base stations $i$ and $j$ and $\delta$ is a constant that depends on the average base-station antenna height and the average mobile antenna height. $C_{i}$ is the region enclosed in the polygon whose sides pass through $b_{i j}$ and are perpendicular to the line connecting $L_{i}$ to $L_{j}$ for $j \in \mathcal{A}_{i}$ (ignoring edge effects).

To find the optimal values of the transmitted pilot-signal powers that will maximize the capacity of the entire network, we calculate the derivatives of the network capacity with respect to $T_{k}$ s and use them in an optimization algorithm. They capture the effect of increases in the transmitted pilot-signal power of one base station on the capacity of the entire network.

The derivative of $n$ with respect to the intercell interference factor $\kappa_{l i}$ is

$$
\frac{\partial n}{\partial \kappa_{l i}}=\frac{-\beta_{i^{*}} \beta_{l} c_{\mathrm{eff}}^{\left(i^{*}\right)}}{\left(\beta_{i^{*}}+\sum_{j=1}^{M} \beta_{j} \kappa_{j i^{*}}\right)^{2}}
$$

if $l \neq i^{*}$ and $i=i^{*}$, and zero otherwise. From (4)

$$
\kappa_{l i}=e^{\left(\gamma \sigma_{s}\right)^{2}} \frac{1}{A_{l}} F_{l i}\left(C_{l}, L_{i}\right)
$$

where

$$
F_{l i}\left(C_{l}, L_{i}\right)=\iint_{C_{l}} \frac{r_{l}^{m}(x, y)}{r_{i}^{m}(x, y)} d A(x, y) .
$$

The region of cell $l, C_{l}$, is a function of the independent variables $L_{l}, L_{p}, T_{l}$, and $T_{p}$, where $p \in \mathcal{A}_{l}$. Thus the partial derivative of $\kappa_{l i}$ with respect to $T_{k}$ is given by

$$
\frac{\partial \kappa_{l i}}{\partial T_{k}}=e^{\left(\gamma \sigma_{s}\right)^{2}}\left[\frac{-1}{A_{l}^{2}} \frac{\partial A_{l}}{\partial T_{k}} F_{l i}\left(C_{l}, L_{i}\right)+\frac{1}{A_{l}} \frac{\partial F_{l i}\left(C_{l}, L_{i}\right)}{\partial T_{k}}\right]
$$

if $l=k$ or $l \in \mathcal{A}_{k}$, and zero otherwise. The intercell interference factors $\kappa_{k i}\left(C_{k}(T), L_{i}\right)$ for $i=1, \ldots, M$, are a function of $T_{k}$. Also, the intercell interference factors $\kappa_{l i}\left(C_{l}(T), L_{i}\right)$ for $l \in$ $\mathcal{A}_{k}$ and $i=1, \ldots, M$, are a function of $T_{k}$. Thus, the derivative of $n$ with respect to the transmitted pilot-signal power $T_{k}$ is

$$
\frac{\partial n}{\partial T_{k}}=\sum_{i=1}^{M} \frac{\partial n}{\partial \kappa_{k i}} \frac{\partial \kappa_{k i}}{\partial T_{k}}+\sum_{i=1}^{M} \sum_{l \in \mathcal{A}_{k}} \frac{\partial n}{\partial \kappa_{l i}} \frac{\partial \kappa_{l i}}{\partial T_{k}}
$$

For the general case, the derivative of $c_{i}$ with respect to $\kappa_{k l}$ is

$$
\frac{\partial c_{i}}{\partial \kappa_{k l}}= \begin{cases}\frac{n_{k} \beta_{k}}{\beta_{l}}, & \text { if } k \neq i \text { and } l=i \\ 0, & \text { otherwise. }\end{cases}
$$

The derivative of $c_{i}$ with respect to $T_{m}$ is

$$
\frac{\partial c_{i}}{\partial T_{m}}=\sum_{k=1}^{M} \frac{\partial c_{i}}{\partial \kappa_{k i}} \frac{\partial \kappa_{k i}}{\partial T_{m}}
$$

The above derivatives will be used in the solution to the optimization problems (32) and (34).

\section{Sensitivity with Respect to Base Station Location}

Another way we can control the intracell and the intercell interferences is by controlling the locations of the base stations. Changing the location of a base station changes the coverage region of that cell and the coverage regions of the adjacent cells. Thus, we wish to find the derivatives of the network capacity with respect to base-station location and use them in an optimization algorithm. They capture the effect of changing the coordinates of one base station $k$ on the capacity of the entire network.

We start by finding the partial derivative of $\kappa_{l i}$ with respect to $L_{k}^{(x)}$

$$
\frac{\partial \kappa_{l i}}{\partial L_{k}^{(x)}}=e^{\left(\gamma \sigma_{s}\right)^{2}}\left[\frac{-1}{A_{l}^{2}} \frac{\partial A_{l}}{\partial L_{k}^{(x)}} F_{l i}\left(C_{l}, L_{i}\right)+\frac{1}{A_{l}} \frac{\partial F_{l i}\left(C_{l}, L_{i}\right)}{\partial L_{k}^{(x)}}\right]
$$

if $l=k$ or $l \in \mathcal{A}_{k}$, and

$$
\frac{\partial \kappa_{l i}}{\partial L_{k}^{(x)}}=e^{\left(\gamma \sigma_{s}\right)^{2}} \frac{1}{A_{l}} \frac{\partial F_{l i}\left(C_{l}, L_{i}\right)}{\partial L_{k}^{(x)}}
$$


if $i=k$ and $l \notin \mathcal{A}_{k}$, and zero otherwise. The intercell interference factors $\kappa_{k i}\left(C_{k}(L), L_{i}\right)$ for $i=1, \ldots, M$, are a function of $L_{k}^{(x)}$. The intercell interference factors $\kappa_{l i}\left(C_{l}(L), L_{i}\right)$ for $l \in \mathcal{A}_{k}$ and $i=1, \ldots, M$, are a function of $L_{k}^{(x)}$. Also, the intercell interference factors $\kappa_{l k}\left(C_{l}, L_{k}\right)$ for $l=1, \ldots, M$ and $l \notin \mathcal{A}_{k}$ are a function of $L_{k}^{(x)}$. Thus, the derivative of the network capacity with respect to the $x$-coordinate of base station $k$ is

$$
\begin{aligned}
\frac{\partial n}{\partial L_{k}^{(x)}}= & \sum_{i=1}^{M} \frac{\partial n}{\partial \kappa_{k i}} \frac{\partial \kappa_{k i}}{\partial L_{k}^{(x)}}+\sum_{i=1}^{M} \sum_{l \in \mathcal{A}_{k}} \frac{\partial n}{\partial \kappa_{l i}} \frac{\partial \kappa_{l i}}{\partial L_{k}^{(x)}} \\
& +\sum_{l=1, l \notin \mathcal{A}_{k}}^{M} \frac{\partial n}{\partial \kappa_{l k}} \frac{\partial \kappa_{l k}}{\partial L_{k}^{(x)}} .
\end{aligned}
$$

We have a similar expression for $L_{k}^{(y)}$.

For the general case, the derivative of $c_{i}$ with respect to $L_{m}^{(x)}$ is

$$
\frac{\partial c_{i}}{\partial L_{m}^{(x)}}=\sum_{k=1}^{M} \frac{\partial c_{i}}{\partial \kappa_{k i}} \frac{\partial \kappa_{k i}}{\partial L_{m}^{(x)}}
$$

The above derivatives will be used in the solution to the optimization problems (33) and (34).

\section{Complexity}

In this section, we evaluate the complexity - the number of multiplications and additions used in the calculations of the relative average intercell interference and the sensitivity analysis. Assume that the coverage region of a cell is approximated by a circle. Let $R_{\text {avg }}$ be the average radius of a cell. Then for an $M$-cell network, the area of the network is proportional to $M R_{\mathrm{avg}}^{2}$. To calculate the integrals in (4) and (5), the network is divided into grid points. The relative interference from a mobile located at the center of that grid point to every base station is calculated. The total relative interference is the sum of the integrals from each grid point. The complexity to calculate the total relative average intercell interference is $O\left(M^{2} R_{\text {avg }}^{2}\right)$.

The complexity to calculate the minimum of $M$ elements is $O(\ln M)$ [25]. Given the intercell interference factors, the complexity to calculate the capacity of the network from (8) is $O\left(M^{2}+\ln M\right)=O\left(M^{2}\right)$. The complexity to calculate the sensitivity with respect to one PCF from (17) is $O(M)$. Let $A_{\text {avg }}$ be the average cardinality of the set of adjacent base stations $\mathcal{A}_{i}$. Then the complexity to calculate the sensitivity with respect to one pilot-signal power from (24) is $O\left(A_{\mathrm{avg}} M^{2}\right)$. The complexity for the calculation of the sensitivity with respect to one base-station location coordinate from (29) is also $O\left(A_{\text {avg }} M^{2}\right)$.

\section{MAXIMIZATION OF CAPACITY}

Having derived the sensitivity of network capacity with respect to PCFs, pilot-signal powers, and base-station locations, we modify the optimization problem (9) to include these pa- rameters. The first optimization problem formed has the PCFs as the independent variables

$$
\begin{array}{ll}
\max _{\underline{n}, \underline{\beta}} & \sum_{i=1}^{M} n_{i} \\
\text { subject to } & 1 \leq \underline{\beta} \leq \underline{\beta}^{\max } \\
& n_{i}+\sum_{j=1}^{M} n_{j} \frac{\beta_{j}}{\beta_{i}} \kappa_{j i}-c_{\mathrm{eff}}^{(i)}\left(\beta_{i}\right) \leq 0, \\
& \text { for } i=1, \ldots, M .
\end{array}
$$

The value of $\beta_{i}^{\max }, i=1, \ldots, M$, should be chosen so that the required transmit power of the mobiles in cell $i$ does not exceed the maximum power that a mobile can transmit. Thus, values of $\beta_{i}^{\max }$ are different and inversely proportional to the radius of cell $i$ since the transmit power of the mobiles is directly proportional to the radius of the cells. The solution for the above optimization problem gives the total network capacity and the optimizing values of the power compensation factors.

We form another optimization problem where the independent variables are the transmitted pilot-signal powers. The optimization problem is

$$
\begin{aligned}
\max _{\underline{n}, \underline{T}} & \sum_{i=1}^{M} n_{i} \\
\text { subject to } & n_{i}+\sum_{j=1}^{M} n_{j} \frac{\beta_{j}}{\beta_{i}} \kappa_{j i}\left(C_{j}, L_{i}\right)-c_{\mathrm{eff}}^{(i)} \leq 0, \\
& \text { for } i=1, \ldots, M .
\end{aligned}
$$

Another possible optimization is for the case when the independent variables are the base-station locations. The optimization problem is

$$
\begin{aligned}
\max _{\underline{n}, \underline{L}} & \sum_{i=1}^{M} n_{i} \\
\text { subject to } & n_{i}+\sum_{j=1}^{M} n_{j} \frac{\beta_{j}}{\beta_{i}} \kappa_{j i}\left(C_{j}, L_{i}\right)-c_{\text {eff }}^{(i)} \leq 0, \\
& \text { for } i=1, \ldots, M .
\end{aligned}
$$

Finally, we maximize the network capacity by optimizing simultaneously the power compensation factors, the transmitted pilot-signal powers, and the base-station locations. The combined optimization problem is

$$
\begin{array}{ll}
\max _{\underline{n}, \underline{\beta}, \underline{\underline{T}} \underline{\underline{L}}} & \sum_{i=1}^{M} n_{i} \\
\text { subject to } & 1 \leq \underline{\beta} \leq \underline{\beta}^{\max } \\
& n_{i}+\sum_{j=1}^{M} n_{j} \frac{\beta_{j}}{\beta_{i}} \kappa_{j i}\left(C_{j}, L_{i}\right)-c_{\mathrm{eff}}^{(i)}\left(\beta_{i}\right) \leq 0 \\
& \text { for } i=1, \ldots, M .
\end{array}
$$

The optimization problems (31)-(34) are mixed integer programming (MIP) problems. The branch-and-bound method is used to arrive at integer solutions for $n_{i}, i=1, \ldots, M$. 
By relaxing the integer variables $n_{i}, i=1, \ldots, M$, to continuous variables, the optimizations in (31)-(34) are solved using a sequential quadratic programming method [27]. In this method, a quadratic programming subproblem is solved at each iteration. An estimate of the Hessian of the Lagrangian is updated at each iteration using the Broyden-Fletcher-Goldfarb-Shanno formula [28]. A line search is performed using a merit function [29]. The quadratic programming subproblem is solved using an active set strategy [30].

In Section VI, we provide results for the optimization problems in (31)-(34) (with the continuous relaxation assumption), which give an upper bound on the optimal value. What is presented is the rounded-down version of the solution. We also solve the MIP problems and provide the number of branches required to arrive at the solution.

We note that unlike (9), (31)-(34) are not convex optimization problems, and so it may be possible for the approaches described above not to converge to a global optimal solution. To ensure that this did not occur, we verified the results of the optimization using simulated annealing (SA) [31], which has many attractive features. In particular, SA can statistically guarantee finding a global optimal solution [32]. On the other hand, it can be quite time consuming to use SA to find an optimal solution, and it is difficult to fine tune. We use an adaptive simulated annealing (ASA) algorithm, which is based on an associated proof that the parameter space can be sampled much more efficiently than by other previous SA algorithms. ${ }^{1}$ Our initial capacity vector used in ASA is the solution returned by the optimization problems (31)-(34). The generating probability density function, the acceptance probability density function, and the cooling temperature schedule used were the default values provided by the algorithm itself.

\section{NUMERICAL RESULTS}

We assume the following for the analysis. The COST-231 propagation model with a carrier frequency of $1800 \mathrm{MHz}$, average base-station height of $30 \mathrm{~m}$, and average mobile height of $1.5 \mathrm{~m}$ is used to determine the coverage region. The path-loss coefficient $m$ is four. The shadow-fading standard deviation $\sigma_{s}$ is $6 \mathrm{~dB}$. The processing gain $W / R$ is $21.1 \mathrm{~dB}$. (This corresponds to the processing gain in IS-95.) The bit energy to interference ratio threshold $\Gamma$ is $9.2 \mathrm{~dB}$. The interference to background noise ratio $I_{0} / N_{0}$ is $10 \mathrm{~dB}$. The voice activity factor $\alpha$ is 0.375 . The whole area is divided into small grids of size 150 by $150 \mathrm{~m}^{2}$.

In what follows, we will study an example with a uniform and nonuniform user distribution in detail and show how the optimization techniques described in this paper, i.e., (9) and (31)-(34), maximize the capacity profile of such a network. The following results have been obtained for the 27-cell CDMA network shown in Fig. 2. The base stations are located at the centers of a hexagonal grid whose radius is $1732 \mathrm{~m}$. Base station 1 is located at the origin. The base stations are numbered consecutively in a spiral pattern. The pilot-signal power of every base station is $1 \mathrm{~W}$.

\footnotetext{
${ }^{1}$ The ASA code and ample documentation are publicly available at
} http://www.ingber.com/

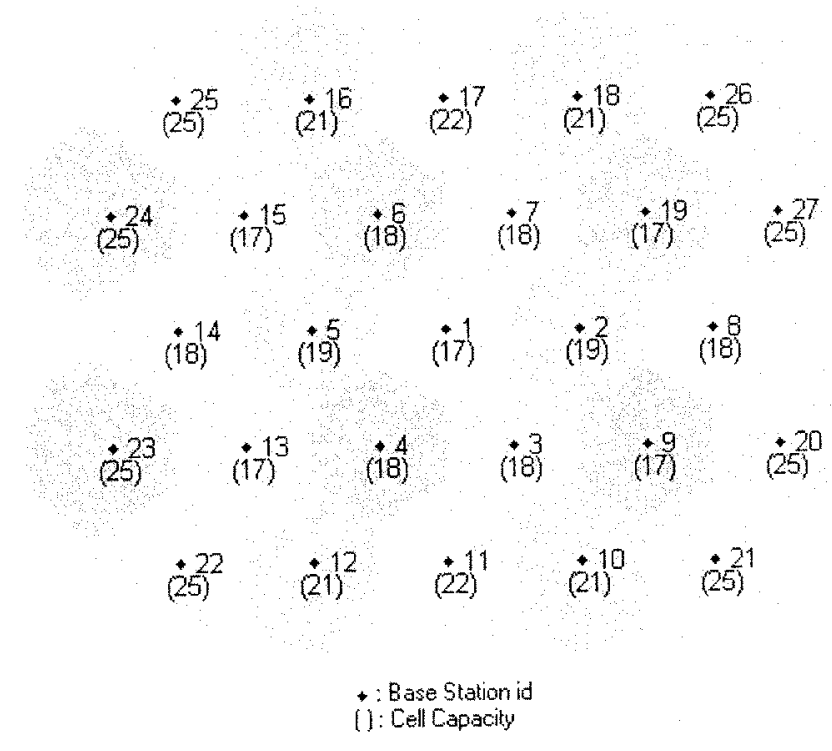

Fig. 2. Capacity in a 27-cell CDMA network with uniform user distribution.

\section{A. Uniform User Distribution}

For a uniform user distribution and power compensation factor equal to one in every cell, the equal capacity of this network calculated from (8) is 18 users per cell, giving a network capacity of 486 . This network capacity becomes 565 if the optimization given in (9) is used. If the capacity of every cell is rounded down to an integer (rounded-down capacity), the capacity of the network is 548. The IP solution of (9) yields a network capacity of 559 (with 56635 branches). The capacities of the individual cells $n_{i}$ are given in parentheses in Fig. 2. It can be seen that for cells on the outer edges of the coverage area, the capacity has increased significantly. This is due to the fact that the intercell interference for these cells is smaller than that for the cells in the interior of the coverage area. Also note that the increase in capacity of the cells on the outer edges increases the intercell interference into their adjacent cells, thereby reducing the capacity of some of those cells from 18 to 17 . The overall network capacity, however, has increased from 486 to 559 .

After running the optimization for PCFs, pilot-signal powers, and base-station locations, i.e., the optimizations described in (31)-(34), the network remains unchanged. Thus, as expected, for a uniform user distribution, a uniform network layout with equal PCFs, equal pilot-signal powers, and equal distances between base stations is optimal. Intuitively, this is not the case for a nonuniform user distribution.

\section{B. Nonuniform User Distribution}

We considered three hot-spot clusters, as shown in Fig. 3. In a cell with a hot spot, the user distribution is no longer uniform. A relative user density assigned to each hot spot specifies how the users in the cell are distributed. The first hot-spot cluster (seven hot spots) is circular in shape. Its center is located at $(-4500$, 2598) $\mathrm{m}$, which coincides with base station 15 and has a radius of $3000 \mathrm{~m}$. The second hot-spot cluster (five hot spots) is rectangular in shape. The lower left corner is at $(-4600,-4200) \mathrm{m}$, and the upper right corner is at $(1400,-1200) \mathrm{m}$. The third hot-spot cluster (four hot spots) is square in shape. The lower 


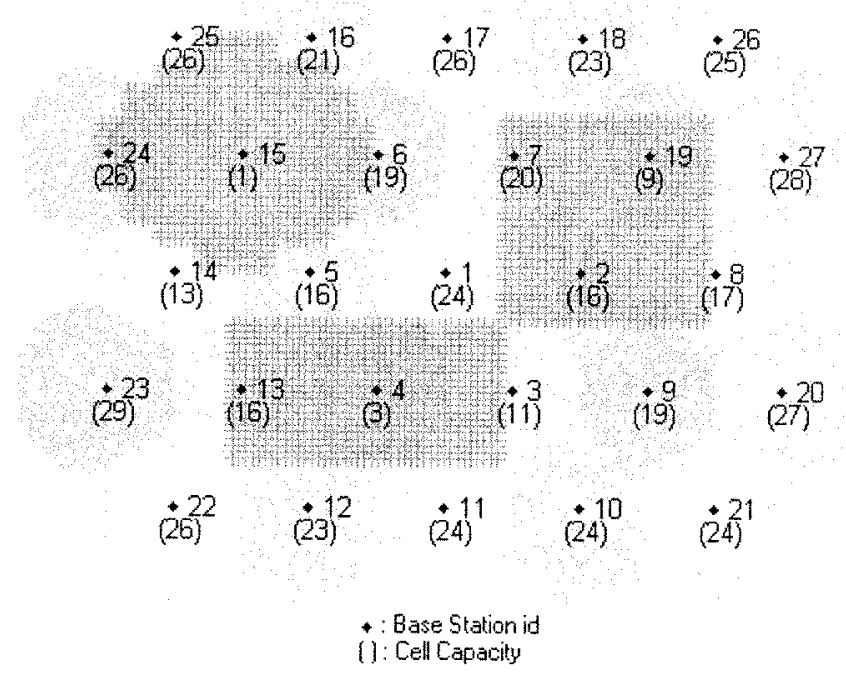

Fig. 3. Capacity in the 27-cell CDMA network with three hot spots.

left corner is at $(1600,-1000) \mathrm{m}$, and the upper right corner is at $(6100,3500) \mathrm{m}$. All the hot spots have the same relative user density per grid point, which is five times that of a grid point with no hot spot.

The equal capacity of this network calculated from (8) is 13 users per cell, giving a network capacity of 351 . The network capacity obtained from (9) is 540 (the rounded-down capacity is 528). The IP solution of (9) yields a network capacity of 536 (with 106610 branches). The capacity in each cell is shown in parentheses in Fig. 3. The capacity of cells 4, 15, and 19, which are inside the hot-spot clusters, has decreased from 18 to 3,17 to 1 , and 17 to 9 , respectively. These cells lose the most capacity due to the increase in intracell and intercell interference because of the nonuniform user distribution. The network capacity optimization tries to increase the sum of the capacities of the cells by adjusting the physical parameters of power compensation factors, pilot-signal powers, and base-station locations. We now examine the advantages of adjusting these parameters in our optimization.

1) Optimization Using Power Compensation Factors: From (31), the maximization of network capacity with respect to PCFs increases the network capacity to 560 (the rounded-down capacity is 546). The MIP solution of (31) yields a network capacity equal to 555 (with 129357 branches) and the cell capacities given in Fig. 4. The values of the optimized PCFs are shown in brackets and the cell capacities are shown in parentheses. After optimization, the capacity of cells 4,15 , and 19 increases from 3 to 12,1 to 9 , and 9 to 14, respectively. Even though the capacity in a few cells has decreased, the smallest capacity in any cell has increased from 1 to 9 . Without the power compensation optimization, the cells with high interference have very small capacity.

The optimization increases the power compensation factors of the cells with high interference. This results in a PCF of 1.64 for cell 4, 1.71 for cell 15 , and 1.56 for cell 19 . Increasing the PCF of a cell increases its signal-to-noise ratio, thus increasing the cell's capacity.

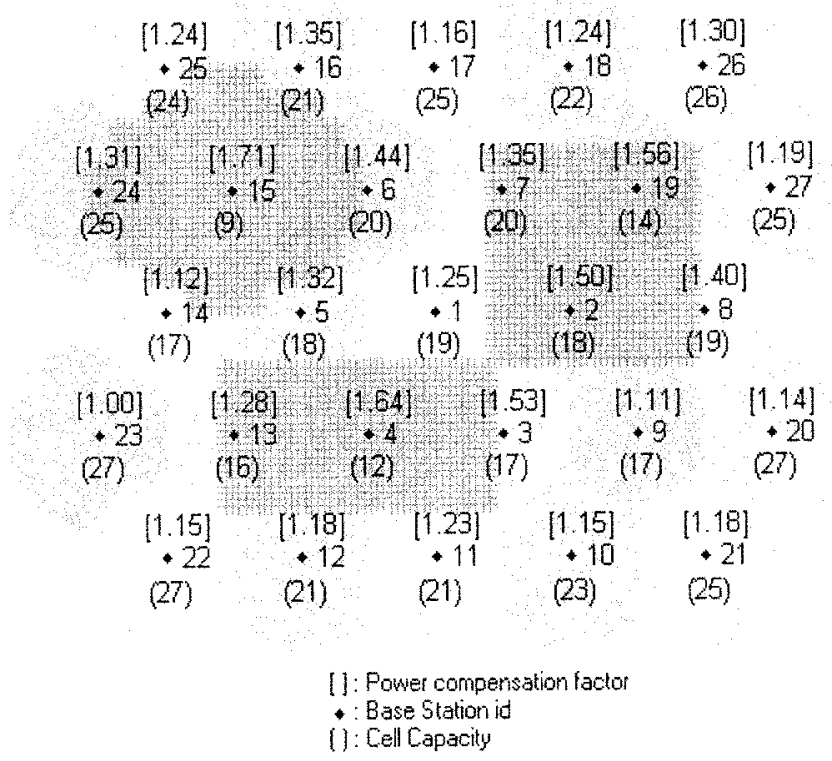

Fig. 4. Capacity in the 27-cell CDMA network, which is optimized using power compensation factors.

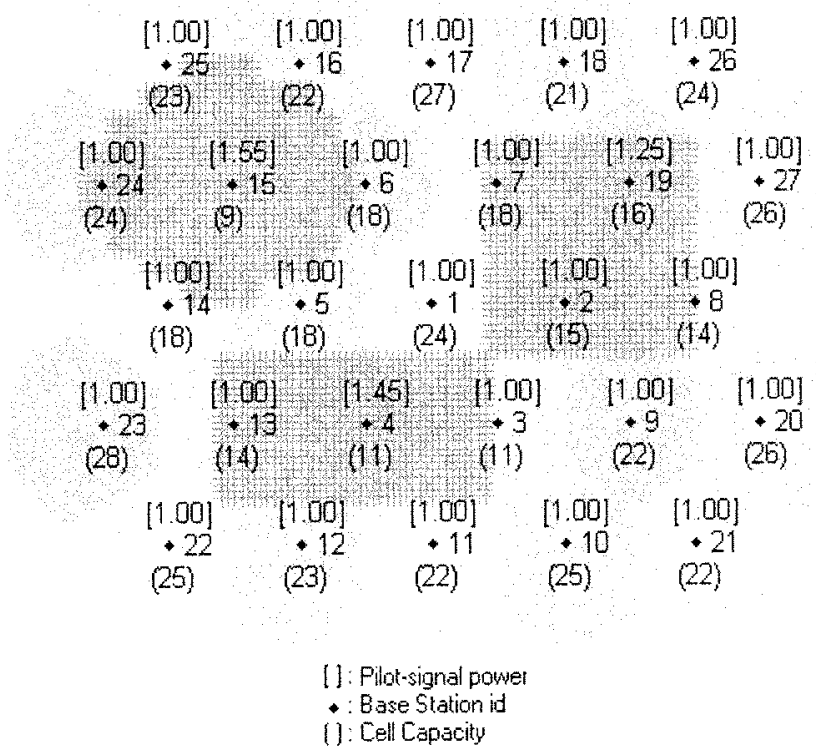

Fig. 5. Capacity in the 27-cell CDMA network, which is optimized using pilot-signal powers.

2) Optimization Using Pilot-Signal Powers: The maximization of network capacity with respect to pilot-signal powers [see (32)] increases the network capacity to 552 (the rounded-down capacity is 539). The MIP solution of (32) yields a network capacity equal to 546 (with 262604 branches) and the cell capacities given in Fig. 5. The values of the optimized pilot-signal powers in watts are shown in brackets, and the capacity is shown in parentheses. After running the optimization, the capacity of cells 4,15 , and 19 increases from 3 to 11,1 to 9 , and 9 to 16 , respectively. The pilot-signal powers of base stations 4,15 , and 19 increase from $1 \mathrm{~W}$ to $1.45,1.55$, and $1.25 \mathrm{~W}$, respectively. This increase in pilot power and thus coverage region does increase 


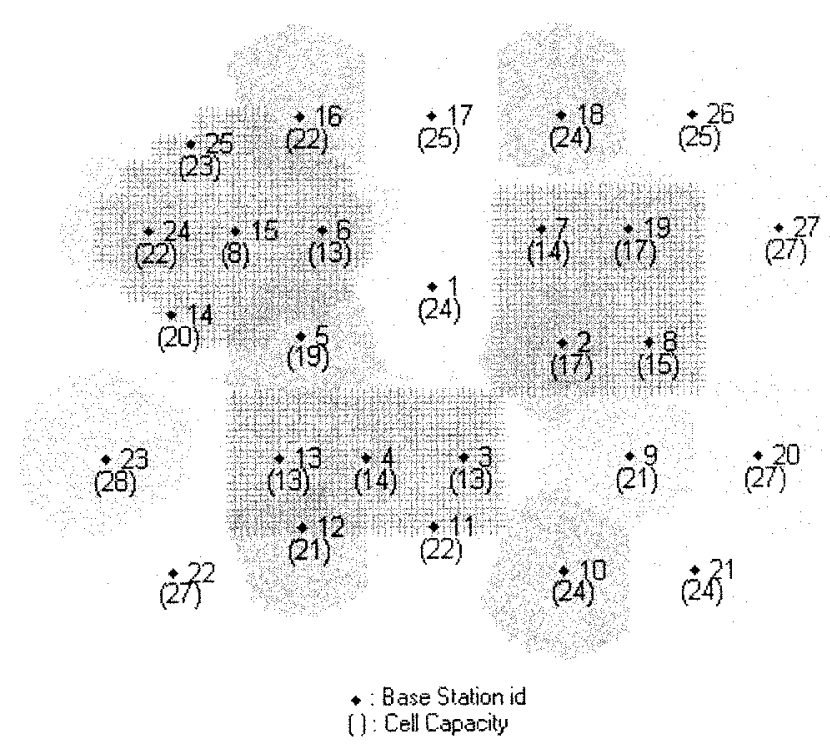

Fig. 6. Capacity in the 27-cell CDMA network, which is optimized using base-station locations.

the intracell interference further, but the intercell interference now decreases more, giving a reduction in total interference. These base stations are essentially taking on the users that have been causing the high intercell interference and controlling their power levels, thus reducing the total interference and resulting in a higher capacity. The optimization has increased the smallest capacity in any cell to nine.

3) Optimization Using Base-Station Locations: The maximization of network capacity with respect to base-station locations [see (33)] produces a network capacity equal to 555 (the rounded-down capacity is equal to 541). The MIP solution of (33) yields a network capacity equal to 549 (with 90194 branches) and the cell capacities given in Fig. 6. The optimization moves base stations $3,13,11$, and 12 closer and places them inside the hot-spot cluster. A similar relocation takes place for base stations $2,7,8$, and 19 that are now placed almost uniformly inside the hot-spot cluster. The high demand area is thus being serviced by more base stations, and the interference to each cell in the hot-spot cluster is comparable. After running the optimization, the capacity of cells 4,15 , and 19 increases from 3 to 14,1 to 8 , and 9 to 17 , respectively. The network capacity increases to 556, and the smallest capacity in any cell increases to eight.

4) Combined Optimization: Finally, we solve (34), which maximizes network capacity by optimizing simultaneously the power compensation factors, pilot-signal powers, and base-station locations. The network capacity increases to 576 (the rounded-down capacity is 560). The MIP solution of (34) yields a network capacity equal to 565 (with 758877 branches) and the cell capacities given in Fig. 7. The optimization increases the smallest cell capacity in the network to 13 . Thus, the optimization achieves the very important goal of increasing the capacity of the individual cells that have very high interference. This results in an increase in total network capacity, and equally important, in an increase in the smallest cell capacity in the network.

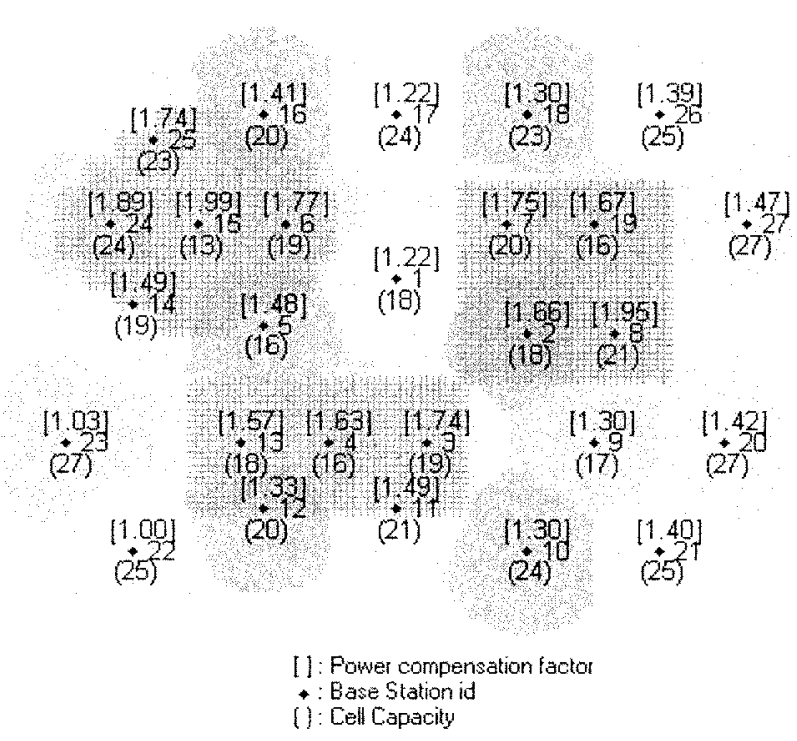

Fig. 7. Capacity in the 27-cell CDMA network, which is optimized using base-station locations, pilot-signal powers, and power compensation factors.

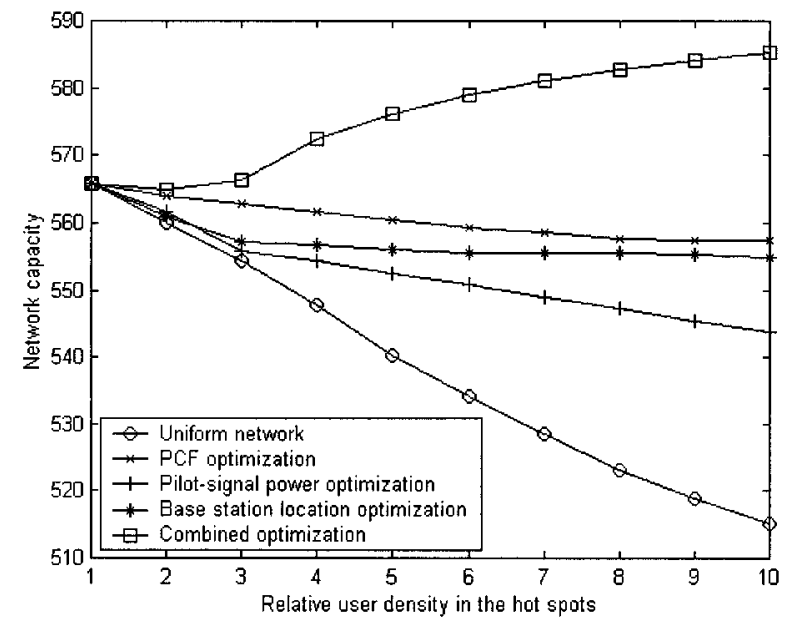

Fig. 8. Network capacity versus relative user density for the 27-cell network.

Fig. 8 summarizes the previous results and presents the values of the network capacity returned from the different optimizations as the relative user density is varied from one to ten. A relative user density of one means no hot spot, i.e., a uniform user distribution. (Recall that the example that was presented in detail in Figs. 3-7 is for a relative user density of five.) Fig. 8 demonstrates clearly the increase in network capacity as a result of optimizing the power compensation factors, the pilot-signal powers, or the base-station locations. It also shows the significant gains in network capacity achieved from the combined optimization approach. In all our examples, the IP/MIP solution improved the rounded-down solution only slightly (around 2\%) but at a cost of considerable computational complexity.

Fig. 9 presents the results of the optimizations for the equal capacity case. The $y$-axis is the capacity in every cell. We see the same trend as in Fig. 8.

The examples above suggest the following observation. For a uniform network layout with a nonuniform user distribution 


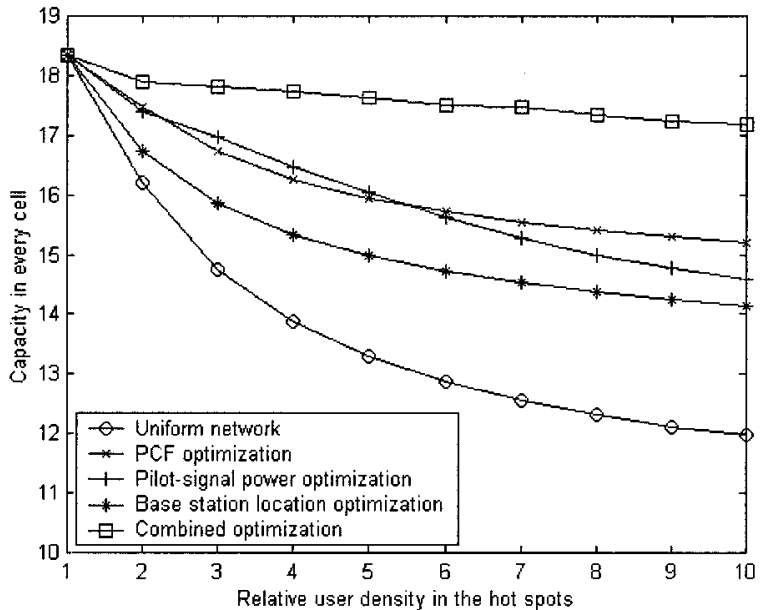

Fig. 9. Capacity in every cell for the equal capacity case versus relative user density for the 27-cell network.

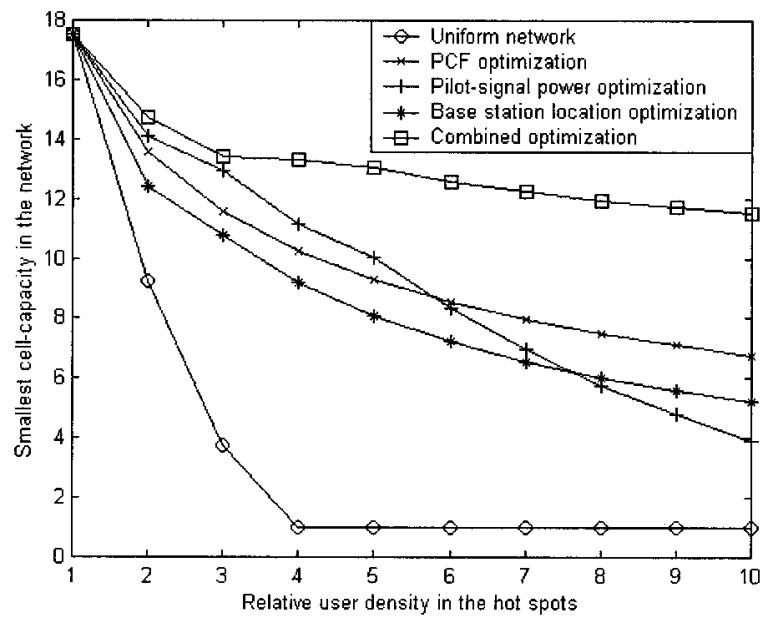

Fig. 10. Smallest cell capacity in the network versus relative user density for the 27-cell network.

and with a relative user density in the hot spots equal to four and higher, the capacity of the cells inside the hot spot clusters drops to one. This is because we are maximizing the total network capacity, i.e., the total number of users in all the cells. Thus, the capacity of a cell inside a hot-spot cluster with a lot of interference is decreased to minimize its effect. This allows for a large increase in the capacities of its neighbors, resulting in the maximization of the total network capacity. This effect is highlighted in Fig. 10, which depicts the smallest cell capacity in the network obtained from optimizations (9) and (31)-(34). Even though the optimizations are not specifically attempting to increase the capacity of any individual cells, the results show a significant increase in the capacity of the cells that have a very small capacity in the uniform network layout case. This motivates a new optimization problem, where we wish to maximize the network capacity while providing a minimum capacity $(\mathrm{mc})$ per cell. In this approach, we first calculate the equal capacity, using (8). We denote this by $n_{\min }$.

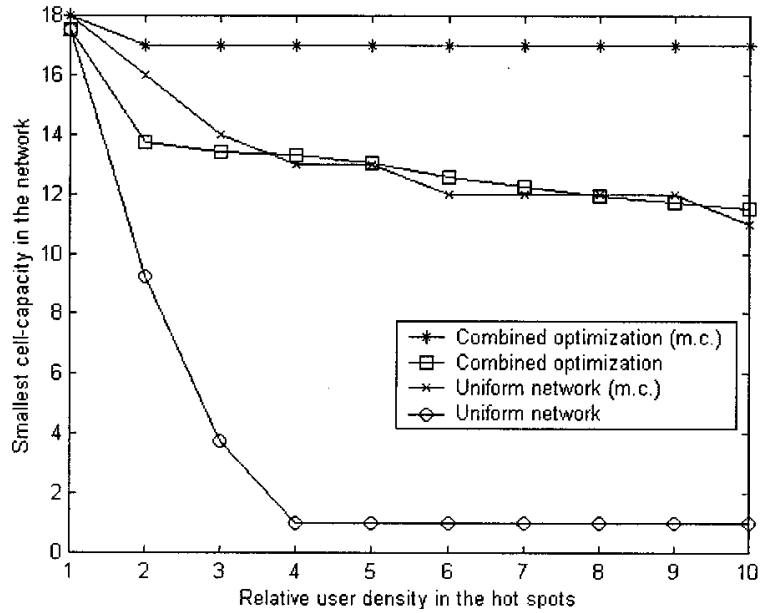

Fig. 11. Smallest cell capacity in the network for the uniform network and combined optimization cases with and without the minimum capacity constraint.

The network capacity is now the solution to the following optimization problem:

$$
\begin{aligned}
\max _{\underline{n}} & \sum_{i=1}^{M} n_{i} \\
\text { subject to } & n_{i}+\sum_{j=1}^{M} n_{i} \kappa_{j i}-c_{\mathrm{eff}} \leq 0, \\
& n_{i} \geq\left\lfloor n_{\min }\right\rfloor, \\
& \text { for } i=1, \ldots, M .
\end{aligned}
$$

In this way, (35) is guaranteed to have a feasible solution and every cell is guaranteed to have a minimum capacity of $\left\lfloor n_{\min }\right\rfloor$. The new combined optimization problem using the power compensation factors, pilot-signal powers, and base-station locations becomes

$$
\begin{aligned}
\max _{\underline{n}, \underline{\beta}, \underline{\underline{T}}, \underline{\underline{L}}} & \sum_{i=1}^{M} n_{i} \\
\text { subject to } & 1 \leq \underline{\beta} \leq \underline{\beta}^{\max } \\
& n_{i}+\sum_{j=1}^{M} n_{j} \frac{\beta_{j}}{\beta_{i}} \kappa_{j i}\left(C_{j}, L_{i}\right)-c_{\mathrm{eff}}^{(i)}\left(\beta_{i}\right) \leq 0 \\
& n_{i} \geq\left\lfloor n_{\min }\right\rfloor \\
& \text { for } i=1, \ldots, M .
\end{aligned}
$$

Fig. 11 compares the smallest capacity in a cell in the network for the four cases of uniform network [uniform network topology with capacity calculated using (9)], combined optimization [optimized network topology and capacity calculated using (34)], uniform network (mc) [uniform network topology and capacity calculated using (35)], and combined optimization (mc) [optimized network topology and capacity calculated using (36)]. Fig. 12 compares the total network capacity for these four cases. Fig. 11 shows that significant improvement can be achieved by imposing the minimum capacity constraint in (35) and (36). In particular, using the combined optimization approach with the minimum capacity constraint, a minimum capacity of 17 users per cell can be achieved. Moreover, as Fig. 12 


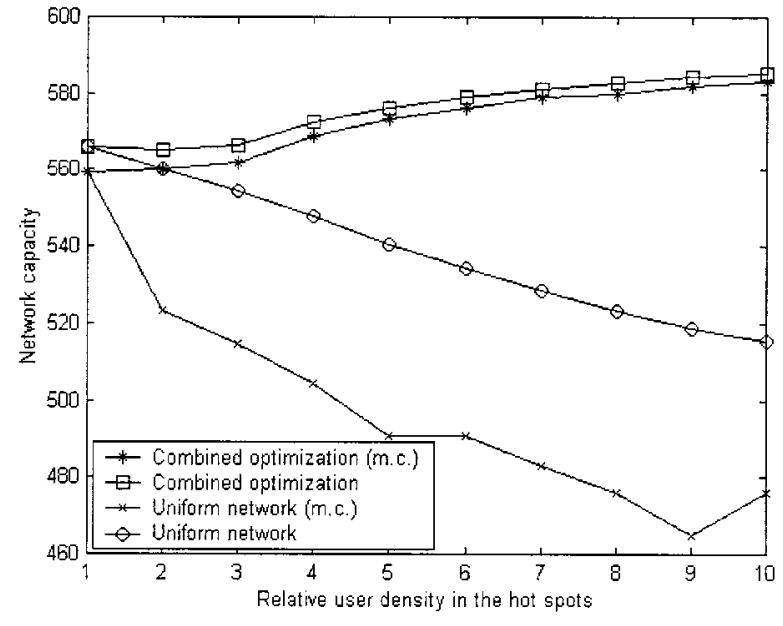

Fig. 12. Network capacity for the uniform network and combined optimization cases with and without the minimum capacity constraint.

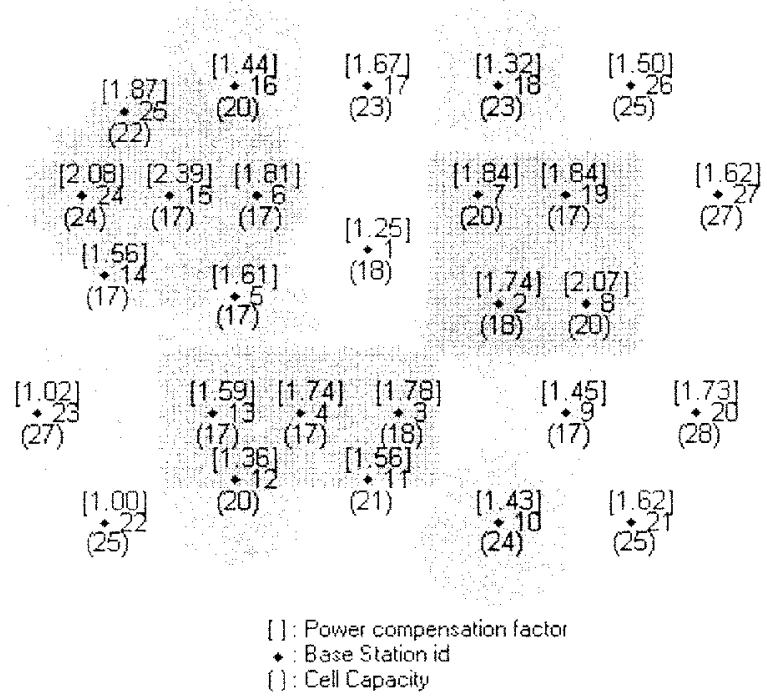

Fig. 13. Capacity in the 27-cell CDMA network, which is optimized using base-station locations, pilot-signal powers, and power compensation factors with a minimum capacity constraint.

shows, this is achieved at a small cost in total network capacity. In fact, for relative user densities larger than four, the difference between the total network capacity from combined optimization and combined optimization (mc) is around three. We would like to point out that, as Fig. 12 also shows, there is a larger drop in total network capacity between the two cases of uniform network and uniform network $(\mathrm{mc})$. Adding the minimum capacity constraint in (35) without the optimization of PCFs, pilot signal powers, and base-station locations causes a larger reduction in the total network capacity. We should also point out that the amount of reduction depends on the number of hot spots in the network. An increase in the number of hot spots and an increase in the relative user density in the hot spots causes a greater reduction in the total network capacity.

Finally, the capacity of the cells for the relative user density of five is given in Fig. 13 in parentheses. The smallest capacity in any cell is 17, as opposed to 13 in Fig. 7. The MIP solution yields a network capacity of 564, which is only one less than the capacity achieved previously using (34). Thus, our results show that maximizing the network capacity, with a minimum capacity constraint, by varying the PCFs, base-station locations, and pilot-signal powers, is the best way to increase capacity in the cells individually and in the network as a whole.

\section{CONCLUSION}

We show how to increase the reverse-link capacity in a CDMA network by varying the transmission power of the mobiles, the pilot-signal powers, and the base-station locations. We calculate the derivative of the reverse-link network capacity with respect to pilot-signal powers, base-station locations, and power compensation factors. These derivatives are then used in an optimization procedure to maximize the network capacity. The results confirm that for a uniform user distribution, a uniform network layout with equal-sized cells is optimal. For a nonuniform distribution, more cells need to be located inside the hot-spot cluster. If pilot-signal power is the only variable parameter, then an increase in pilot-signal powers of congested cells increases network capacity. Even though the intracell interference increases, a greater reduction in intercell interference is achieved, which yields an increase in the overall capacity. We also construct and solve constrained optimization problems, which guarantee a minimum capacity for every individual cell while maximizing the total network capacity. These results indicate that including a hard constraint on the minimum capacity of individual cells has little effect on network capacity given the flexibility of optimizing the transmission power of the mobiles, the pilot-signal powers, and the location of the base stations. However, without such flexibility, the hard constraint on cell capacity imposes a significant penalty on network capacity. The network design technique introduced accommodates postdeployment design changes in response to changes in demand, particularly by changing the PCFs and the pilot-signal powers.

\section{REFERENCES}

[1] K. S. Gilhousen, I. M. Jacobs, R. Padovani, A. J. Viterbi, L. A. Weaver, and C. E. Wheatley, "On the capacity of a cellular CDMA system," IEEE Trans. Veh. Technol., vol. 40, pp. 303-312, May 1991.

[2] A. J. Viterbi, CDMA Principles of Spread Spectrum Communication. Reading, MA: Addison-Wesley, 1995.

[3] R. Padovani, "Reverse link performance of IS-95 based cellular systems," IEEE Personal Commun., vol. 1, no. 3, pp. 28-34, 1994.

[4] A. M. Viterbi and A. J. Viterbi, "Erlang capacity of a power controlled CDMA system," IEEE J. Select. Areas Commun., vol. 11, pp. 892-900, Aug. 1993.

[5] A. J. Viterbi, A. M. Viterbi, K. S. Gilhousen, and E. Zehavi, "Soft handoff extends CDMA cell coverage and increases reverse link capacity," IEEE J. Select. Areas Commun., vol. 12, pp. 1281-1288, Oct. 1994.

[6] A. Baiocchi, F. Sestini, and F. Priscoli, "Effects of user mobility on the capacity of a CDMA network," Eur. Trans. Telecommun., vol. 7, no. 4, pp. 305-314, July/Aug. 1996.

[7] Y. Ishikawa and N. Umeda, "Capacity design and performance of call admission control in cellular CDMA systems," IEEE J. Select. Areas Commun., vol. 15, pp. 1627-1635, Oct. 1997.

[8] K. Takeo and S. Sato, "The proposal of CDMA cell design scheme considering change in traffic distributions," in Proc. IEEE Int. Symp. Spread Spectrum Techniques and Applications, vol. 1, Sept. 1998, pp. 229-233.

[9] K. Takeo and S. Sato, "Evaluation of a CDMA cell design algorithm considering nonuniformity of traffic and base station locations," IEICE Trans. Fundamentals, vol. E81-A, no. 7, pp. 1367-1377, July 1998. 
[10] S. Shin, C. Cho, and D. Sung, "Interference-based channel assignment for DS-CDMA cellular systems," IEEE Trans. Veh. Technol., vol. 48, pp. 233-239, Jan. 1999.

[11] C. U. Saraydar and A. Yener, "Capacity enhancement for CDMA systems through adaptive cell sectorization," in Proc. IEEE Wireless Communications and Networking Conf., Sept. 1999, pp. 1139-1143.

[12] J. S. Evans and D. Everitt, "On the teletraffic capacity of CDMA cellular networks," IEEE Trans. Veh. Technol., vol. 48, pp. 153-165, Jan. 1999.

[13] M. Soleimanipour and G. H. Freeman, "A realistic approach to the capacity of cellular CDMA systems," in Proc. IEEE Vehicular Technology Conf., vol. 2, 1996, pp. 1125-1129.

[14] J. Yang and W. C. Y. Lee, "Design aspects and system evaluation of IS-95 based CDMA systems," in Proc. IEEE Int. Conf. Universal Personal Communications, Oct. 1997, pp. 381-385.

[15] "Mobile station-base station compatibility standard for dual-mode wideband spread spectrum cellular system,", Tech. Rep. IS-95-A, May 1995.

[16] T. Suzuki, K. Takeo, M. Nishino, and Y. Amezawa, "Microcell quality control scheme for PCS CDMA systems considering nonuniform traffic distribution," in Proc. IEEE Int. Conf. Universal Personal Communications, 1993, pp. 239-243.

[17] T. Dohi, M. Sawahashi, and F. Adachi, "Performance of SIR based power control in the presence of nonuniform traffic distribution," in Proc. IEEE Int. Conf. Universal Personal Communications, Nov. 1995, pp. 334-338.

[18] S. Seo, T. Dohi, and F. Adachi, "SIR-Based transmit power control of reverse link for coherent DS-CDMA mobile radio," IEICE Trans. Commun., vol. E81-B, no. 7, pp. 1508-1516, July 1998.

[19] R. G. Akl, M. V. Hegde, M. Naraghi-Pour, and P. S. Min, "Flexible allocation of capacity in multi-cell CDMA networks," in Proc. IEEE Vehicular Technology Conf., vol. 2, May 1999, pp. 1643-1647.

[20] H. Susuki, "A statistical model for urban radio propagation," IEEE Trans. Commun., vol. 25, pp. 673-680, July 1997.

[21] R. G. Akl, M. V. Hegde, A. Chandra, and P. S. Min, "CCAP: CDMA capacity allocation and planning," Washington Univ., Tech. Rep., Apr. 1998.

[22] D. Everitt, "Analytic traffic models of CDMA cellular networks," in Proc. 14th Int. Teletraffic Congr., June 1994, pp. 349-356.

[23] G. L. Nemhauser and L. A. Wolsey, Integer and Combinatorial Optimization. New York: Wiley, 1988.

[24] M. S. Bazaraa and J. J. Jarvis, Linear Programming and Network Flows. New York: Wiley, 1977.

[25] T. S. Rappaport, Wireless Communications Principles and Practice. Englewood Cliffs, NJ: Prentice-Hall, 1996.

[26] T. H. Cormen, C. E. Leiserson, and R. L. Rivest, Introduction to Algorithms. New York: McGraw-Hill, 1992.

[27] M. J. D. Powell, "A fast algorithm for nonlinearly constrained optimization calculations," Numer. Anal., vol. 630, 1978.

[28] T. F. Coleman and Y. Li, "An interior, trust region approach for nonlinear minimization subject to bounds," SIAM J. Optimiz., vol. 6, pp. 418-445, 1996.

[29] S. P. Han, "A globally convergent method for nonlinear programming," J. Optimiz. Theory Applicat., vol. 22, p. 297, 1977.

[30] P. E. Gill, W. Murray, and M. H. Wright, Practical Optimization. New York: Academic, 1981

[31] S. Kirkpatrick, C. D. Gelatt, and M. P. Vecchi, "Optimization by simulated annealing," Science, vol. 220, no. 4593, pp. 671-680, 1983.

[32] L. Ingber, "Simulated annealing: Practive versus theory," J. Math. Computat. Modeling, vol. 18, no. 11, pp. 29-57, 1993.
Robert G. Akl (S'93-M'00) received the B.S. degree in computer science in 1994 and the B.S., M.S., and D.Sc. degrees in electrical engineering from Washington University, St. Louis, MO, in 1994, 1996, and 2000, respectively.

He is currently a System's Engineer with Comspace Corporation, Coppell, TX. His interests are in the design and analysis of wireless communications networks.

Manju V. Hegde (S'84-M'87) received the B.Tech. degree in electrical engineering from the Indian Institute of Technology, Bombay, in 1979, the M.M.S. degree in finance from the University of Bombay, India, in 1981, the M.S. degree in statistics from the University of Toledo, Toledo, OH, in 1983, and the $\mathrm{Ph} . \mathrm{D}$. degree in computer, information and control engineering from the University of Michigan, Ann Arbor, in 1987.

From May 1981 to August 1982, he was a Consultant for 20th Century Consultancy and Financial Services Pvt. Ltd. From 1987 to 1997, he was with the Department of Electrical and Computer Engineering, Louisiana State University, Baton Rouge, as an Assistant Professor and then Associate Professor. Since 1997, he has been an Associate Professor in the Electrical Engineering Department, Washington University, St. Louis, MO. Currently, he is on leave of absence from Washington University and with Celox Networks, Inc., St. Louis, an Internet equipment provider. His research interests include communication and information theory, telecommunication networks, control, and signaling in packet networks.

Prof. Hegde received the Outstanding Graduate Student Award in Computer, Information and Control Engineering from the University of Michigan in 1987.

Mort Naraghi-Pour (S'81-M'84) was born in Tehran, Iran, on May 15, 1954. He received the B.S.E. degree from Tehran University in 1977 and the M.S. and $\mathrm{Ph} . \mathrm{D}$. degrees in electrical engineering from the University of Michigan, Ann Arbor, in 1983 and 1987, respectively.

During 1978, he was a student at the Philips International Institute, Eindhoven, The Netherlands, where he also did research with the telecommunication switching group of the Philips Research Laboratories. Since August 1987, he has been with the Department of Electrical and Computer Engineering, Louisiana State University (LSU), Baton Rouge, where he is an Associate Professor. His research and teaching interests are in digital communication, computer networks, wireless networks, information theory, and coding. He is currently on leave from LSU working as a Senior Member of Technical Staff at Celox Networks, St. Louis, MO

Paul S. Min (S'85-M'87-SM'95) received the B.S., M.S., and Ph.D. degrees in electrical engineering from the University of Michigan, Ann Arbor, in 1982, 1984 , and 1987, respectively.

He was a Member of Technical Staff at Bellcore during 1987-1990. He joined the Department of Electrical Engineering, Washington University, St. Louis, MO, in 1990, where he currently is an Associate Professor. 